\title{
Resolutions of De Concini-Procesi ideals of hooks
}

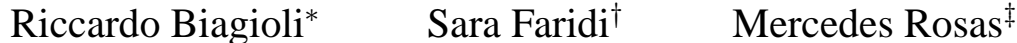

\begin{abstract}
We find a minimal generating set for the defining ideal of the schematic intersection of the set of diagonal matrices with the closure of the conjugacy class of a nilpotent matrix indexed by a hook partition. The structure of this ideal allows us to compute its minimal free resolution and give an explicit description of the graded Betti numbers, and study its Hilbert series and regularity.
\end{abstract}

\section{Introduction}

A nilpotent matrix of size $n$, over a field $k$ of characteristic 0 , can be labelled with a partition of $n$, say $\lambda=\left(\lambda_{1}, \lambda_{2}, \ldots, \lambda_{\ell}\right)$, where the $\lambda_{i}$ are the sizes of its Jordan blocks; let $\mathcal{O}_{\lambda}$ denote the conjugacy class of such a matrix. The problem of finding a generating set for the defining ideal of the Zariski closure of $\mathcal{O}_{\lambda}$ was suggested by De Concini and Procesi [DP], and many authors since then conjectured or proved various generating sets (Eisenbud and Saltman [ES], Tanisaki [T], and Weyman [W]).

The schematic intersection of $\overline{\mathcal{O}}_{\lambda^{\prime}}$ (where $\lambda^{\prime}$ denotes the conjugate of $\lambda$ ), with the set of all diagonal matrices, whose defining ideal we denote by $\mathcal{I}_{\lambda}$, has also been studied by the authors mentioned above. In this case, however, the generating set is simpler to understand. De Concini and Procesi [DP] produced a generating set for $\mathcal{I}_{\lambda}$, and proved that the quotient of the polynomial ring $R=k\left[x_{1}, \ldots, x_{n}\right]$ by $\mathcal{I}_{\lambda}$, is isomorphic to the cohomology ring of a certain subvariety of the flag variety. Moreover, as a representation of the symmetric group $\mathfrak{S}_{n}, R / \mathcal{I}_{\lambda}$ is isomorphic to the induction of the trivial representation of the Young subgroup $\mathfrak{S}_{\lambda_{1}} \times \cdots \times \mathfrak{S}_{\lambda_{\ell}}$.

Garsia and Procesi [GP] studied the graded character of this representation, and showed that it could be expressed in terms of Kostka-Foulkes polynomials, leading the way to more investigations in this subject by Aval and Bergeron [AB], Bergeron and Garsia [BG], among others. These investigations, were facilitated by Tanisaki's work [T], where a simpler generating set for $\mathcal{I}_{\lambda}$, in terms of elementary partially symmetric functions, is defined.

In this paper we study the ideals $\mathcal{I}_{\lambda}$, which we call De Concini-Procesi ideals. We reduce Tanisaki's generating set in the case when $\lambda$ is a hook partition, and obtain a minimal generating set. This reduced generating set allows us to compute the bigraded Poincaré series associated to such an ideal (i.e. the generating function encoding the ranks of the free modules appearing in a minimal free resolution of the ideal), by using only relatively basic algebraic techniques. We also give a description of the Hilbert series of $R / \mathcal{I}_{\lambda}$. We end the paper with a couple of combinatorial results. We compute the generating function of the single Poincaré series, and a combinatorial recurrence for the bigraded one.

This paper is organized as follows. In Section 2 contains a review of resolutions, Cohen-Macaulay rings, and the other commutative algebra tools that we use in the paper. Section 3 , we give the basic definitions of partitions and the language used in the paper. We then introduce De Concini-Procesi ideals, and compute a new generating set for them in the case of hooks; we show later in Section 5 that this generating set is minimal. In Section 4 we study the resolutions of such ideals, and conclude with the formula of the corresponding bigraded Poincaré series. Finally, in Section 5 we compute the regularity and we give an explicit formula for the Hilbert series of the module $R / \mathcal{I}_{\lambda}$.

\footnotetext{
* Institut Camille Jordan, Université Claude Bernard Lyon 1, Villeurbanne, FRANCE, biagioli@math.univ-lyon1.fr

$\dagger$ Department of Mathematics, Dalhousie University, Halifax, CANADA, faridi@ mathstat.dal.ca (research supported by NSERC)

${ }_{\ddagger}^{\ddagger}$ Department of Mathematics and Statistics, York University, York, CANADA, rosas@ mathstat.yorku.ca
} 
Weyman and Shimozono have brought to our attention that a resolution for $\mathcal{I}_{\lambda}$ can be obtained also with a different technique, namely by using Lascoux resolution and the Koszul complex. Some details on this construction can be found in [W].

Acknowledgments: All the test examples that supported this research were run using the computer algebra program Macaulay2 [GS]. We would like to thanks François Bergeron, Emmanuel Briand, Tony Geramita and the referee for useful comments, and Mark Shimozono and Jerzy Weyman for telling us about resolutions of nilpotent closures, and many helpful remarks and suggestions.

\section{Commutative algebra tools}

Let $R=k\left[x_{1}, \ldots, x_{n}\right]$ be a polynomial ring over a field $k$ of characteristic 0 , with the standard grading $\operatorname{deg} x_{i}=1$, for all $i$. Let $\mathfrak{m}=\left(x_{1}, \ldots, x_{n}\right)$ be the (irrelevant) homogeneous maximal ideal of $R$. We are interested in the quotient $S=R / I$ where $I$ is an ideal of $R$ generated by homogeneous polynomials.

Definition 2.1 (Minimal free resolution). A free resolution of $R / I$ is an exact complex $\mathbb{F}$

$$
0 \longrightarrow \cdots \stackrel{\delta_{i+1}}{\longrightarrow} F_{i} \stackrel{\delta_{i}}{\longrightarrow} F_{i-1} \stackrel{\delta_{i-1}}{\longrightarrow} \cdots \stackrel{\delta_{2}}{\longrightarrow} F_{1} \stackrel{\delta_{1}}{\longrightarrow} R \stackrel{\delta_{0}}{\longrightarrow} R / I \longrightarrow 0 .
$$

of free $R$-modules $F_{i}\left(F_{0}=R\right)$. The resolution is minimal if $\delta_{i}\left(F_{i}\right) \subseteq \mathfrak{m} F_{i-1}$ for $i>0$.

If each $F_{i}$ is a free module of rank $\beta_{i}$, the $\beta_{i}$ are called the Betti numbers of $R / I$; these are independent of which minimal resolution one considers.

In the case where $I$ is a homogeneous ideal, and therefore $R / I$ is graded, we define the graded Betti numbers of $R / I$. This is done by making the maps $\delta_{i}$ homogeneous, so that they take a degree $j$ element of $F_{i}$ to a degree $j$ element of $F_{i-1}$. To serve this purpose the degree of each generator of $F_{i}$ is adjusted. So we can write the free module $F_{i}=R^{\beta_{i}}$ as $R^{\beta_{i}}=\bigoplus_{j} R(-j)^{\beta_{i, j}}$ where for a given integer $a, R(a)$ is the same as $R$ but with a new grading: $R(a)_{d}=R_{a+d}$. So the resolution shown in above becomes

$$
0 \longrightarrow \bigoplus_{j} R(-j)^{\beta_{m, j}} \stackrel{\delta_{m}}{\longrightarrow} \bigoplus_{j} R(-j)^{\beta_{m-1, j}} \stackrel{\delta_{m-1}}{\longrightarrow} \cdots \stackrel{\delta_{2}}{\longrightarrow} \bigoplus_{j} R(-j)^{\beta_{1, j}} \stackrel{\delta_{1}}{\longrightarrow} R \stackrel{\delta_{0}}{\longrightarrow} R / I \longrightarrow 0 .
$$

This is called the graded minimal free resolution of $R / I$, and the $\beta_{i, j}$ are the graded Betti numbers of $R / I$. Clearly, $\sum_{j} \beta_{i, j}=\beta_{i}$.

Definition 2.2 (Bigraded Poincaré series). The bigraded Poincaré series of an ideal $I$ is the generating function for the graded Betti numbers of $I$ :

$$
P_{R / I}(q, t)=\sum_{i, j} \beta_{i, j} q^{i} t^{j}
$$

Definition 2.3 (Linear resolution). The graded resolution described in (1) is a linear resolution, if for some $u, \beta_{i, j}=0$ unless $j=u+i-1$.

Discussion 2.4 (Resolutions using mapping cones). The mapping cone technique provides a way to build a free resolution of an ideal by adding generators one at a time. A resolution obtained using mapping cones is not in general minimal. However, we will be focusing only on the special case of multiplication by a nonzerodivisor, in which case we obtain a minimal free resolution.

Suppose that $I$ is an ideal in the polynomial ring $R$, and $e \in \mathfrak{m}$ is a nonzerodivisor in $R / I$ (i.e. $e$ is a regular element $\bmod I$ ). The goal is to build a minimal free resolution of $R /(I+(e))$ starting from a minimal free resolution of $R / I$. Consider the short exact sequence

$$
0 \longrightarrow R /(I:(e)) \stackrel{\cdot e}{\longrightarrow} R / I \longrightarrow R /(I+(e)) \longrightarrow 0
$$


where $I:(e)$ is the quotient ideal consisting of all elements $x \in R$ such that $x e \in I$. Since $e$ is a nonzerodivisor in $R / I$, we have $I:(e)=I$, and so our short exact sequence turns into

$$
0 \longrightarrow R / I \stackrel{\cdot e}{\longrightarrow} R / I \longrightarrow R /(I+(e)) \longrightarrow 0 .
$$

Suppose we have a minimal free resolution of $R / I$

$$
0 \longrightarrow \cdots \stackrel{\delta_{i+1}}{\longrightarrow} A_{i} \stackrel{\delta_{i}}{\longrightarrow} A_{i-1} \stackrel{\delta_{i-1}}{\longrightarrow} \cdots \stackrel{\delta_{2}}{\longrightarrow} A_{1} \stackrel{\delta_{1}}{\longrightarrow} R \stackrel{\delta_{0}}{\longrightarrow} R / I \longrightarrow 0 .
$$

Then we can obtain the following minimal free resolution of $R /(I+(e))$

$$
0 \longrightarrow \cdots \stackrel{d_{i+1}}{\longrightarrow} F_{i} \stackrel{d_{i}}{\longrightarrow} F_{i-1} \stackrel{d_{i-1}}{\longrightarrow} \cdots \stackrel{d_{2}}{\longrightarrow} F_{1} \stackrel{d_{1}}{\longrightarrow} R \stackrel{d_{0}}{\longrightarrow} R /(I+(e)) \longrightarrow 0
$$

where for each $i>0$, as a free $R$-module

$$
F_{i}=A_{i} \oplus A_{i-1} \text { and } d_{i}(x, y)=\left(e y+\delta_{i}(x),-\delta_{i-1}(y)\right) .
$$

We now focus on the grading of each $F_{i}$. Suppose that the element $e \in R$ is homogeneous of degree $m$, and for each $i$, each of the free modules $A_{i}$ in (2) are of the form

$$
A_{i}=\bigoplus_{j} R(-j)^{\beta_{i, j}}
$$

where the $\beta_{i, j}$ are the graded Betti numbers. We would like to compute the graded Betti numbers of $R /(I+$ $(e))$. Below we give an explicit description of the grading for each $F_{i}$; the gist of the argument, which can be found in Schenck's book [Sc], is that we need to twist the graded resolution of $R / I$ in (2) by $m$ to obtain a resolution of $R /(I:(e))$ that make the maps that produce the mapping cone resolution homogeneous. So each $A_{i-1}$-component of $F_{i}$ is a twist of $A_{i-1}$ appearing in (2).

Lemma 2.5. Consider the minimal free resolutions (2) of $R / I$, and (3) of $R /(I+(e))$ obtained by mapping cones. For each $i>0$ we have

$$
F_{i}=\bigoplus_{j} R(-j)^{\beta_{i, j}} \oplus \bigoplus_{j} R(-j-m)^{\beta_{i-1, j}} .
$$

Proof. In the case where $i=1$, we have the homogeneous map $d_{1}: A_{1} \oplus R \longrightarrow R$ where $d_{1}(x, y)=$ $e y+\delta_{1}(x)$. In particular, if $x \in A_{1}$ is a homogeneous element of degree $t$, then $d_{1}(x, 0)=\delta_{1}(x)$ is also a degree $t$ homogeneous element of $R$. If $y \in R$ is a homogeneous element of degree $t$, then $d_{1}(0, y)=e y$ has degree $t+m$. In order to make $d_{1}$ a homogeneous (degree 0 ) map, we shift the grading of the component $R$ of $F_{i}$ by $m$, so that

$$
F_{1}=\bigoplus_{j} R(-j)^{\beta_{1, j}} \oplus R(-m) .
$$

The same argument applies, by induction, to each step $i$ of the resolution.

Corollary 2.6. Let $I$ be an ideal of the polynomial ring $R$ and $e \in \mathfrak{m}$ be a homogeneous element of degree $m$ which is a nonzerodivisor in $R / I$. Then

$$
P_{R /(I+(e))}(q, t)=\left(1+q t^{m}\right) P_{R / I}(q, t) .
$$

Proof. By Lemma 2.5, if for a fixed $i, A_{i}=\bigoplus_{j=0}^{b_{i}} R(-j)^{\beta_{i, j}}$ then

$$
F_{i}=\bigoplus_{j=0}^{b_{i}} R(-j)^{\beta_{i, j}} \oplus \bigoplus_{j=0}^{b_{i-1}} R(-j-m)^{\beta_{i-1, j}}
$$


So we have

$$
\begin{aligned}
P_{R /(I+(e))}(q, t) & =1+\sum_{i \geq 1}\left(\sum_{j=0}^{b_{i}} \beta_{i, j} t^{j}+\sum_{j=0}^{b_{i-1}} \beta_{i-1, j} t^{j+m}\right) q^{i} \\
& =\sum_{i \geq 0} \sum_{j=0}^{b_{i}} \beta_{i, j} t^{j} q^{i}+t^{m} \sum_{i \geq 0} \sum_{j=0}^{b_{i}} \beta_{i, j} t^{j} q^{i+1} \\
& =\left(1+q t^{m}\right) \sum_{i \geq 0} \sum_{j=0}^{b_{i}} \beta_{i, j} t^{j} q^{i}=\left(1+q t^{m}\right) P_{R / I}(q, t) .
\end{aligned}
$$

Recall that a (square-free) monomial ideal is an ideal generated by (square-free) monomials in the variables $x_{1}, \ldots, x_{n}$. If $I$ and $J$ are two ideals of $R$, their quotient is the ideal defined as

$$
I: J=\{x \in R \mid x J \subseteq I\} .
$$

Definition 2.7 (linear quotients). If $I \subset k\left[x_{1}, \ldots, x_{n}\right]$ is a monomial ideal and $G(I)$ is its unique minimal set of monomial generators, then $I$ is said to have linear quotients if there is an ordering $M_{1}, \ldots, M_{m}$ on the elements of $G(I)$ such that for every $i=2, \ldots, m$, the quotient ideal

$$
\left(M_{1}, \ldots, M_{i-1}\right): M_{i}
$$

is generated by a subset of the variables $x_{1}, \ldots, x_{n}$.

Lemma 2.8. Let $I$ be an ideal in the polynomial ring $R=k\left[x_{1}, \ldots, x_{n}\right]$ generated by all square-free monomials of a fixed degree $m$. Then

\section{I has linear quotients;}

2. $R / I$ has a linear resolution;

\section{3. $R / I$ is Cohen-Macaulay.}

Proof. Statements (1) and (3) follow from [HH]. Statement (2) is true because of the Eagon-Reiner [ER] criterion for Cohen-Macaulayness of square-free monomial ideals, and the fact that the Alexander dual of $I$ is also generated by all square-free monomials of a fixed degree.

\section{De Concini-Procesi Ideals}

We now introduce a family of ideals $\left\{\mathcal{I}_{\lambda}\right\}_{\lambda}$ of the polynomial ring $R=k\left[x_{1}, \ldots, x_{n}\right]$ indexed by partitions $\lambda$ of $n$. These ideals were first introduced by De Concini-Procesi in [DP]. They showed that for any partition $\lambda$ of $n, R / \mathcal{I}_{\lambda}$ is the coordinate ring of the diagonal matrices which are in the closure of the conjugacy class of a nilpotent matrix of Jordan block structure given by the partition $\lambda^{\prime}$, the conjugate of $\lambda$. We start with some definitions and notation about partitions, that will be used in the rest of this paper.

We let $\mathbb{N}^{+}=\{1,2, \ldots\}$, and $\mathbb{N}=\mathbb{N}^{+} \cup\{0\}$. The cardinality of a set $S$ is denoted by $|S|$. We define a partition of $n \in \mathbb{N}$ to be a finite sequence $\lambda=\left(\lambda_{1}, \ldots, \lambda_{k}\right) \in \mathbb{N}^{k}$, such that $\sum_{i=1}^{k} \lambda_{i}=n$ and $\lambda_{1} \geq \ldots \geq \lambda_{k}$. If $\lambda$ is a partition of $n$ we write $\lambda \vdash n$. The nonzero terms $\lambda_{i}$ are called parts of $\lambda$. The number of parts of $\lambda$ is called the length of $\lambda$, denoted by $\ell(\lambda)$.

The Young diagram of a partition $\left(\lambda_{1}, \ldots, \lambda_{k}\right) \vdash n$, is the diagram with $\lambda_{i}$ squares in the $i^{\text {th }}$-row. We use the symbol $\lambda$ for both a partition and its associated Young diagram. For example, the diagram of $\lambda=$ $(5,4,2,1)$ is illustrated in Figure 1.

For a partition $\lambda=\left(\lambda_{1}, \ldots, \lambda_{k}\right)$ denote the conjugate partition $\lambda^{\prime}:=\left(\lambda_{1}^{\prime}, \ldots, \lambda_{h}^{\prime}\right)$, where for each $i \geq 1$, $\lambda_{i}^{\prime}$ is the number of parts of $\lambda$ that are bigger than or equal to $i$. The diagram of $\lambda^{\prime}$ is obtained by flipping the diagram of $\lambda$ across the diagonal. 


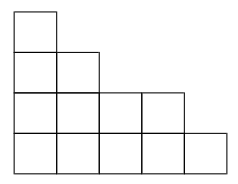

Figure 1: The partition $\lambda=(5,4,2,1)$

A partition is said to be a hook if it is of the form $\lambda=\left(a, 1^{b}\right)$, with $a, b \in \mathbb{N}$. It will often be useful to denote hook partitions using a different notation. The hook $\lambda=\left(a+1,1^{b}\right)$ in Frobenius's notation [M, page $3]$ will be denoted by $\lambda=(a \mid b)$. Note that its conjugate is $\lambda^{\prime}=(b \mid a)$.

From now on, we shall assume that a partition of $n$ has $n$ terms. So we will add enough zero terms to any partition until we have the right number of terms. Let $\lambda=\left(\lambda_{1}, \ldots, \lambda_{n}\right)$ be a partition of $n$, and $\lambda^{\prime}=\left(\lambda_{1}^{\prime} \ldots, \lambda_{n}^{\prime}\right)$ its conjugate partition. For any $1 \leq k \leq n$, we define

$$
\delta_{k}(\lambda):=\lambda_{n}^{\prime}+\lambda_{n-1}^{\prime}+\ldots+\lambda_{n-k+1}^{\prime} .
$$

Recall that for any $1 \leq r \leq n$, the elementary symmetric polynomial $[\mathrm{M}]$ is defined by

$$
e_{r}\left(x_{1}, \ldots, x_{n}\right):=\sum_{1 \leq i_{1}<\ldots<i_{r} \leq n} x_{i_{1}} x_{i_{2}} \cdots x_{i_{r}} .
$$

Given a subset $S \subseteq\left\{x_{1}, \ldots, x_{n}\right\}$, let $e_{r}(S)$ be the $r^{\text {th }}$ elementary symmetric polynomial in the variables in $S$. Clearly, every $e_{r}(S)$ is a homogeneous polynomial in $R$ of degree $r$.

We are now ready to introduce the ideals originally defined by De Concini and Procesi [DP]. We use a different and simpler set of generators with respect to the original one, which was defined by Tanisaki [T].

Definition 3.1 (De Concini-Procesi ideal). We let $\mathcal{C}_{\lambda}$ denote the collection of partial elementary symmetric polynomials

$$
\mathcal{C}_{\lambda}=\left\{e_{r}(S)\left|S \subseteq\left\{x_{1}, \ldots, x_{n}\right\},\right| S \mid=k \geq 1, k \geq r>k-\delta_{k}(\lambda)\right\} .
$$

The De Concini-Procesi ideal $\mathcal{I}_{\lambda}$ is the homogeneous ideal generated by the elements of $\mathcal{C}_{\lambda}$, in symbols,

$$
\mathcal{I}_{\lambda}:=\left(\mathcal{C}_{\lambda}\right) .
$$

Example 3.2. Let $\lambda=(3,1,0,0) \vdash 4$ and $\lambda^{\prime}=(2,1,1,0)$. Then $\left(\delta_{1}(\lambda), \ldots, \delta_{4}(\lambda)\right)=(0,1,2,4)$. Hence $\left(1-\delta_{1}(\lambda), \ldots, 4-\delta_{4}(\lambda)\right)=(1,1,1,0)$, and the collection $\mathcal{C}_{\lambda}$ consists of the following elements. For $k=1$ there is no admissible $e_{r}(S)$. For $k=2$ we get the set of monomials:

$$
x_{1} x_{2}, x_{1} x_{3}, x_{1} x_{4}, x_{2} x_{3}, x_{2} x_{4}, x_{3} x_{4} .
$$

For $k=3$, we get

$$
\begin{gathered}
x_{1} x_{2}+x_{1} x_{3}+x_{2} x_{3}, x_{1} x_{2}+x_{1} x_{4}+x_{2} x_{4}, x_{1} x_{3}+x_{1} x_{4}+x_{3} x_{4}, x_{2} x_{3}+x_{2} x_{4}+x_{3} x_{4} \\
x_{1} x_{2} x_{3}, x_{1} x_{2} x_{4}, x_{1} x_{3} x_{4}, x_{2} x_{3} x_{4} .
\end{gathered}
$$

Finally for $k=4$, we get the complete set of the elementary symmetric functions $e_{r}\left(x_{1}, x_{2}, x_{3}, x_{4}\right)$, for $1 \leq r \leq 4$.

Remark 3.3. Note that $\delta_{n}(\lambda)=n$, for any partition $\lambda$ of $n$. Hence when we set $k=n$ in (4), we obtain that $\mathcal{I}_{\lambda}$ contains the ideal generated by the elementary symmetric polynomials in all the variables. It is well known that $e_{1}\left(x_{1}, \ldots, x_{n}\right), \ldots, e_{n}\left(x_{1}, \ldots, x_{n}\right)$ are algebraically independent (this is due to Gauss; see [M]), and hence they form a regular sequence over $R$. Therefore $R / \mathcal{I}_{\lambda}$ is an Artinian ring. result.

When the indexing partition $\lambda$ is a hook, the ideal $\mathcal{I}_{\lambda}$ can be split in two parts. We have the following 
Proposition 3.4 (A reduced generating set for hook partitions). Let $\lambda=(a \mid b) \vdash n$ be a hook. Then the ideal associated to $\lambda$ in the polynomial ring $k\left[x_{1}, \ldots, x_{n}\right]$ is

$$
\mathcal{I}_{\lambda}=\mathcal{M}_{b+1}+\mathcal{E}_{b}
$$

where

$$
\mathcal{M}_{b+1}=\left(x_{i_{1}} \cdots x_{i_{b+1}} \mid 1 \leq i_{1}<\ldots<i_{b+1} \leq n\right)
$$

is the ideal generated by all square-free monomials in $x_{1}, \ldots, x_{n}$ of degree $b+1$, and

$$
\mathcal{E}_{b}=\left(e_{i}\left(x_{1}, \ldots, x_{n}\right) \mid 1 \leq i \leq b\right)
$$

is the ideal generated by all elementary symmetric polynomials of degree $\leq b$ in the variables $x_{1}, \ldots, x_{n}$.

Proof. The partition $\lambda=(a \mid b)$ is of size $n=a+b+1$. We can write

$$
\lambda^{\prime}=(b \mid a)=(b+1, \underbrace{1, \ldots, 1}_{a}, \underbrace{0, \ldots, 0}_{b}) .
$$

Then we have

$$
\left(\delta_{1}(\lambda), \delta_{2}(\lambda), \ldots, \delta_{n}(\lambda)\right)=(\underbrace{0, \ldots, 0}_{b}, 1,2, \ldots, a, n)
$$

and so

$$
\left(1-\delta_{1}(\lambda), 2-\delta_{2}(\lambda), \ldots, n-\delta_{n}(\lambda)\right)=(1,2,3, \ldots, b, \underbrace{b, \ldots, b}_{a}, 0) .
$$

The definition of $\mathcal{C}_{\lambda}$ in (4) implies that no $k$, with $1 \leq k \leq b$, contributes a generator to the ideal $\mathcal{I}_{\lambda}$.

The first index making a nontrivial contribution to the set $\mathcal{C}_{\lambda}$ is $k=b+1$, which adds to $\mathcal{C}_{\lambda}$ all $e_{b+1}(S)$, with $|S|=b+1$, or in other words all the square-free monomials of degree $b+1$ in the variables $x_{1}, \ldots, x_{n}$. We denote by $\mathcal{M}_{b+1}$ the ideal generated by these square-free monomials.

Now all the indices $k$, with $b+2 \leq k \leq n-1$ add to $\mathcal{C}_{\lambda}$ elements of the form $e_{r}(S)$, with $k \geq r \geq b+1$, and $|S|=k$. Each such $e_{r}(S)$ is a homogeneous polynomial of degree $r$, which we can write as the sum of square-free monomials of degree $r$. Since $r \geq b+1$, and all square-free monomials of degree $b+1$ or more are already in $\mathcal{I}_{\lambda}$, such $e_{r}(S)$ do not contribute any new generators to $\mathcal{I}_{\lambda}$.

Finally, for $k=n$ we obtain all the elementary symmetric polynomials in all the variables. For the same reasons as above, the only new contributions are

$$
e_{1}\left(x_{1}, \ldots, x_{n}\right), e_{2}\left(x_{1}, \ldots, x_{n}\right), \ldots, e_{b}\left(x_{1}, \ldots, x_{n}\right) .
$$

We denote the ideal generated by these elementary symmetric polynomials by $\mathcal{E}_{b}$. We conclude that $\mathcal{I}_{\lambda}=$ $\mathcal{M}_{b+1}+\mathcal{E}_{b}$.

Example 3.5. Let $\lambda=(2 \mid 1) \vdash 4$. It follows from the computations in Example 3.2, that the ideal $\mathcal{I}_{\lambda}$ splits into two parts

$$
\mathcal{I}_{\lambda}=\left(x_{1} x_{2}, x_{1} x_{3}, x_{1} x_{4}, x_{2} x_{3}, x_{2} x_{4}, x_{3} x_{4}\right)+\left(x_{1}+x_{2}+x_{3}+x_{4}\right) .
$$

The first part is generated by all monomials of degree 2 in the variables $x_{1}, x_{2}, x_{3}, x_{4}$, and the second is generated by $e_{1}\left(x_{1}, x_{2}, x_{3}, x_{4}\right)$, the elementary symmetric polynomial of degree 1 .

For hooks, the reduced generating set described in Proposition 3.4 is much smaller than that described in Definition 3.1 (it is in fact minimal), and hence simpler to understand. In the rest of the paper, we use this presentation of $\mathcal{I}_{\lambda}$ to describe the Betti numbers and other numerical information of the algebra $R / \mathcal{I}_{\lambda}$. 


\section{Bigraded Poincaré series of De Concini-Procesi ideals of hooks}

In this section we study the minimal free resolutions of the De Concini-Procesi ideal $\mathcal{I}_{\lambda}$ of a hook $\lambda=(a \mid b)$. We have seen that $\mathcal{I}_{\lambda}$ is the sum of two ideals

$$
\mathcal{I}_{\lambda}=\mathcal{M}_{b+1}+\mathcal{E}_{b}
$$

where $\mathcal{M}_{b+1}$ is generated by monomials, and $\mathcal{E}_{b}$ is generated by elementary symmetric functions. Below we show how we can recover the resolution of $\mathcal{I}_{\lambda}$ using the resolutions of each one of the summands.

Since $\mathcal{M}_{b+1}$ is generated by all square-free monomials of $R=k\left[x_{1}, \ldots, x_{n}\right]$ that have degree $b+1$, by Lemma 2.8, $\mathcal{M}_{b+1}$ is a Cohen-Macaulay ideal with linear resolutions and linear quotients. On the other hand, it is easy to see that all the minimal primes of $\mathcal{M}_{b+1}$ have uniform height $n-b$. This is because every generator of $\mathcal{M}_{b+1}$ is a product of exactly $b+1$ variables in the set $\left\{x_{1}, \ldots, x_{n}\right\}$, and so a minimal subset of $\left\{x_{1}, \ldots, x_{n}\right\}$ that shares at least one variable with each one of these generators must have $n-b$ elements. Such an ideal will have height equal to $n-b$, and so it follows that $\operatorname{dim} R / \mathcal{M}_{b+1}=b$.

We have thus shown that

Corollary 4.1. For a hook $\lambda=(a \mid b)$, the ideal $\mathcal{M}_{b+1}$ of $R$ has linear quotients, linear resolution, and $R / \mathcal{M}_{b+1}$ is Cohen-Macaulay of (Krull) dimension $b$.

Remark 4.2. Let $G\left(\mathcal{M}_{b+1}\right)$ denote the minimal monomial generating set for $\mathcal{M}_{b+1}$. We can arrange the elements of $G\left(\mathcal{M}_{b+1}\right)$ in descending lexicographic order as $M_{1}, \ldots, M_{m}$. Take such a monomial $M_{i}=$ $x_{j_{1}} \cdots x_{j_{b+1}}$, written so that $j_{1}<j_{2}<\ldots<j_{b+1}$. Since $\left(M_{1}, \ldots, M_{i-1}\right)$ is a monomial ideal, and $M_{i}$ is also a monomial, the quotient ideal $\left(M_{1}, \ldots, M_{i-1}\right): M_{i}$ is generated by monomials. Observe that

1. If $s<j_{t}$ for some $j_{t} \in\left\{j_{1}, \ldots, j_{b+1}\right\}$ and $s \notin\left\{j_{1}, \ldots, j_{b+1}\right\}$, then $x_{s} \in\left(M_{1}, \ldots, M_{i-1}\right): M_{i}$.

This is because the monomial $\frac{x_{s} M_{i}}{x_{j_{t}}}$ is a degree $b+1$ monomial that is lexicographically larger than $M_{i}$, that is, $\frac{x_{s} M_{i}}{x_{j_{t}}} \in\left\{M_{1}, \ldots, M_{i-1}\right\}$.

2. If $u$ is a monomial in $\left(M_{1}, \ldots, M_{i-1}\right): M_{i}$, then $M_{l} \mid u M_{i}$ for some $l<i$. Since $M_{l}>_{\text {lex }} M_{i}$, there exists $x_{s}$, such that $x_{s} \mid M_{l}, x_{s} \nmid M_{i}$ and $s<j_{t}$ for some $j_{t} \in\left\{j_{1}, \ldots, j_{b+1}\right\}$.

It follows that $x_{s} \mid u$, and $\left(M_{1}, \ldots, M_{i-1}\right): M_{i}$ is generated by the set of variables $x_{s}$, with $s<j_{b+1}$ and $s \notin\left\{j_{1}, \ldots, j_{b+1}\right\}$ as described in part 1 . This proves that $\mathcal{M}_{b+1}$ has linear quotients.

Next, we focus on the ideal $\mathcal{E}_{b}$, which is generated by the first $b$ elementary symmetric functions. As observed in Remark 3.3, $\mathcal{I}_{\lambda}$ contains the regular sequence $e_{1}\left(x_{1}, \ldots, x_{n}\right), \ldots, e_{n}\left(x_{1}, \ldots, x_{n}\right)$, and hence $R / \mathcal{I}_{\lambda}$ is of (Krull) dimension 0 .

Proposition 4.3. For a hook $\lambda=(a \mid b)$, the set of generators

$$
e_{1}\left(x_{1}, \ldots, x_{n}\right), \ldots, e_{b}\left(x_{1}, \ldots, x_{n}\right)
$$

of $\mathcal{E}_{b}$ form a regular sequence over the quotient ring $R / \mathcal{M}_{b+1}$.

Proof. Let $S=R / \mathcal{M}_{b+1}$. We know by Corollary 4.1 that $S$ is a Cohen-Macaulay ring, and $\operatorname{dim} S=b$. To show that $e_{1}\left(x_{1}, \ldots, x_{n}\right), \ldots, e_{b}\left(x_{1}, \ldots, x_{n}\right)$ forms a regular sequence in $S$, by Theorem 2.1.2 of [BH], it is enough to show that $\operatorname{dim} S / \mathcal{E}_{b}=0$. Now, $S / \mathcal{E}_{b}=R / \mathcal{I}_{\lambda}$, and the latter is an Artinian ring, and hence of dimension 0 .

We are now ready to state our central claim.

Theorem 4.4 (Main theorem). Let $\lambda=(a \mid b)$ be a hook. Then the bigraded Poincaré series for the ideal $\mathcal{I}_{\lambda}$ is the following

$$
P_{R / \mathcal{I}_{\lambda}}(q, t)=\prod_{k=1}^{b}\left(1+q t^{k}\right) \cdot\left(1+q t^{b+1} \sum_{i=0}^{a}\left(\begin{array}{c}
b+i \\
b
\end{array}\right)(1+q t)^{i}\right) .
$$


Proof. As usual, let $\mathcal{I}_{\lambda}=\mathcal{M}_{b+1}+\mathcal{E}_{b}$.

Step 1. The ideal $\mathcal{M}_{b+1}$ has linear quotients (Corollary 4.1). It follows from Corollary 1.6 of [HT] that the bigraded Poincaré series of $\mathcal{M}_{b+1}$ is the following:

$$
P_{R / \mathcal{M}_{b+1}}(q, t)=1+\sum_{M \in G\left(\mathcal{M}_{b+1}\right)}(1+q t)^{|\operatorname{set}(M)|} q t^{\operatorname{deg}(M)}
$$

where, if we arrange the elements of $G\left(\mathcal{M}_{b+1}\right)$ in descending lexicographic order as $M_{1}, \ldots, M_{m}$, then for $i=1, \ldots, m$

$$
\operatorname{set}\left(M_{i}\right)=\left\{j \in\{1, \ldots, n\} \mid x_{j} \in\left(M_{1}, \ldots, M_{i-1}\right): M_{i}\right\} .
$$

As the degree of each of the monomials generating $\mathcal{M}_{b+1}$ is $b+1$, Equation (8) turns into

$$
P_{R / \mathcal{M}_{b+1}}(q, t)=1+q t^{b+1} \sum_{M \in G\left(\mathcal{M}_{b+1}\right)}(1+q t)^{|\operatorname{set}(M)|} .
$$

If $M_{i}=x_{i_{1}} \cdots x_{i_{b+1}}$, by Remark 4.2

$$
\operatorname{set}\left(M_{i}\right)=\left\{u \leq i_{b+1} \mid x_{u} \nmid M_{i}\right\},
$$

so

$$
\left|\operatorname{set}\left(M_{i}\right)\right|=i_{b+1}-(b+1) .
$$

We have shown that, if $M$ is any degree $b+1$ square-free monomial with highest index $u$ (that is, $x_{u} \mid M$ and $x_{v} \nmid M$ for $v>u$ ), then $|\operatorname{set}(M)|=u-(b+1)$. So to compute the sum in (9), all we have to do is count the number of square-free degree $b+1$ monomials with highest index $u$, for any given $u$. This number is clearly $\left(\begin{array}{c}u-1 \\ b\end{array}\right)$. So for a given $i$, the number of degree $b+1$ square-free monomials $M$ with $|\operatorname{set}(M)|=i$ is exactly $\left(\begin{array}{c}b+i \\ b\end{array}\right)$.

Therefore, $P_{R / \mathcal{M}_{b+1}}(q, t)$ is equal to

$$
1+q t^{b+1} \sum_{i=0}^{n-b-1}\left(\begin{array}{c}
b+i \\
b
\end{array}\right)(1+q t)^{i}=1+q t^{b+1} \sum_{i=0}^{a}\left(\begin{array}{c}
b+i \\
b
\end{array}\right)(1+q t)^{i}
$$

since by Equation (10), $i$ can reach at most $n-b-1$, which by definition is equal to $a$.

Step 2. Since $\mathcal{E}_{b}$ is generated by a regular sequence over $R / \mathcal{M}_{b+1}$ (Proposition 4.3), we can use a mapping cone construction to find its minimal graded resolution (see Discussion 2.4). We do this by adding the generators of $\mathcal{E}_{b}$, one at a time, to $\mathcal{M}_{b+1}$, and applying Corollary 2.6. As the generators $e_{1}\left(x_{1}, \ldots, x_{n}\right), \ldots, e_{b}\left(x_{1}, \ldots, x_{n}\right)$ of $\mathcal{E}_{b}$ have degrees $1, \ldots, b$, respectively, each time we add a $e_{i}\left(x_{1}, \ldots, x_{n}\right)$, the Poincaré series gets multiplied by a factor of $\left(1+q t^{i}\right)$, and hence from (11) we obtain that $P_{R / \mathcal{I}_{\lambda}}(q, t)$ equals

$$
\prod_{k=1}^{b}\left(1+q t^{k}\right) \cdot P_{R / \mathcal{M}_{b+1}}(q, t)=\prod_{k=1}^{b}\left(1+q t^{k}\right) \cdot\left(1+q t^{b+1} \sum_{i=0}^{a}\left(\begin{array}{c}
b+i \\
b
\end{array}\right)(1+q t)^{i}\right) .
$$




\section{Some consequences of the Main Theorem}

We study some of the consequences of our main theorem. We prove that our new generating set is indeed minimal.

Corollary 5.1 (The set of generators of $\mathcal{I}_{\lambda}$ is minimal). Let $\lambda=(a \mid b)$ be a hook. The generating set for $\mathcal{I}_{\lambda}$ described in Proposition 3.4 is minimal.

Proof. The cardinality of the generating set of $\mathcal{I}_{\lambda}$ described in Proposition 3.4 is $\left(\begin{array}{c}n \\ b+1\end{array}\right)+b$. On the other hand, the minimal number of generators of $\mathcal{I}_{\lambda}$ is the first Betti number $\beta_{1}$ of $R / \mathcal{I}_{\lambda}$, which is the coefficient of $q$ in the Poincaré series $P_{R / \mathcal{I}_{\lambda}}(q, 1)$. It is easy to see by Theorem 4.4 that this coefficient is

$$
b+1+\sum_{i=1}^{a}\left(\begin{array}{c}
b+i \\
b
\end{array}\right) \text {. }
$$

So all we have to show is that $\left(\begin{array}{c}n \\ b+1\end{array}\right)+b=b+1+\sum_{i=1}^{a}\left(\begin{array}{c}b+i \\ b\end{array}\right)$ which is equivalent to showing that

$$
\left(\begin{array}{c}
n \\
b+1
\end{array}\right)=\sum_{i=0}^{n-b-1}\left(\begin{array}{c}
b+i \\
b
\end{array}\right)
$$

This last equation follows easily from induction on $n$.

\section{Regularity of Hooks}

Definition 5.2 (Castelnuovo-Mumford regularity). Let $I$ be an ideal of a $R=k\left[x_{1}, \ldots, x_{n}\right]$. The CastelnuovoMumford regularity or simply regularity of $R / I$, denoted by $\operatorname{reg}(R / I)$ is defined as the maximum value of of $j-i$ where the graded Betti number $\beta_{i, j} \neq 0$ in a minimal free resolution of $R / I$.

Corollary 5.3 (Regularity of hooks). Let $\lambda=(a \mid b)$ be a hook. Then $\operatorname{reg}(R / I)=b(b+1) / 2$.

Proof. The graded Betti numbers $\beta_{i, j}$ appear as the coefficients of the Poincaré series

$$
P_{R / \mathcal{I}_{\lambda}}(q, t)=\underbrace{\prod_{k=1}^{b}\left(1+q t^{k}\right)}_{\text {Factor } 1} \cdot \underbrace{\left(1+q t^{b+1} \sum_{i=0}^{a}\left(\begin{array}{c}
b+i \\
b
\end{array}\right)(1+q t)^{i}\right)}_{\text {Factor } 2} .
$$

So the question is to find the term $q^{i} t^{j}$ in this polynomial, where the coefficient $\beta_{i, j}$ is nonzero and $j-i$ is maximum. The terms with nonzero coefficients in each factor are of the following forms:

Factor 1: $q^{m} t^{b_{1}+\ldots+b_{m}}$ where $1 \leq b_{1}<\ldots<b_{m} \leq b, 0 \leq m \leq b$, Factor 2: $q^{e+1} t^{e+b+1} \quad$ where $0 \leq e \leq a$.

To show that $\operatorname{reg}(R / I)=\frac{b(b+1)}{2}$, we need to show that this bound is achieved by the possible choices of $j-i$, and is the maximum possible bound. Consider the terms in Factor 1. We have

$$
\begin{aligned}
b_{1}+\ldots+b_{m}-m & \leq((b-(m-1))+(b-(m-2))+\ldots+b)-m \\
& =((1+2+\ldots+b)-(1+2+\ldots+(b-m)))-m \leq \frac{b(b+1)}{2}-b .
\end{aligned}
$$

Similarly, for terms in Factor 2, since $b \geq 0$, we have $e+b+1-(e+1)=b$. Hence, for the product of a term in Factor 1 and a term in Factor 2 we have

$$
b_{1}+\ldots+b_{m}+e+b+1-(m+e+1) \leq \frac{b(b+1)}{2} .
$$

The bound is achieved if $m=b$, so that $b_{1}=1, \ldots, b_{m}=b$, and for any $e$, so that we have the term with nonzero coefficient

$$
q^{e+b+1} t^{(1+\ldots+b)+e+b+1}=q^{e+b+1} t^{\frac{b(b+1)}{2}+e+b+1}
$$

which clearly has the property that $j-i=\frac{b(b+1)}{2}$, as desired. 
Remark 5.4 (Hooks are level algebras). We note that for a hook $\lambda=(a \mid b), R / \mathcal{I}_{\lambda}$ is a level algebra. Indeed, since $R / \mathcal{I}_{\lambda}$ is Artinian, its projective dimension is $n$ (or observe that the highest power of $q$ appearing in Equation (7) is $n$ ), which is the same as the height of the ideal $\mathcal{I}_{\lambda}$. Moreover, the coefficient of $q^{n}$ in this equation is

$$
\left(\begin{array}{c}
b+a \\
b
\end{array}\right) t^{n+\frac{b(b+1)}{2}} .
$$

This implies that $R / \mathcal{I}_{\lambda}$ is level of type $\left(\begin{array}{c}b+a \\ b\end{array}\right)$ with socle degree $\frac{b(b+1)}{2}$ (see, for example, [GL, Definition 0.2]). The regularity that we find above is therefore the expected value.

\section{The Hilbert series of hooks}

We finish this section with the computation of the Hilbert series of $R / \mathcal{I}_{\lambda}$ when $\lambda$ is a hook partition, namely, the series

$$
h_{R / \mathcal{I}_{\lambda}}(q)=\sum_{s=0}^{\infty} \operatorname{dim}_{k}\left(R / \mathcal{I}_{\lambda}\right)_{s} q^{s}
$$

where as usual $\operatorname{dim}_{k}$ means dimension as a vector space over $k$. This has been done in the general case of a partition $\lambda=\left(\lambda_{1}, \ldots, \lambda_{n}\right)$ of $n$ by Garsia and Procesi. In [GP], they provide an explicit basis for $R / \mathcal{I}_{\lambda}$ as a $\mathbb{Q}$-module, from which it follows that $\operatorname{dim}_{k}\left(R / \mathcal{I}_{\lambda}\right)=\left(\begin{array}{c}n \\ \lambda_{1}, \ldots, \lambda_{n}\end{array}\right)$. and

$$
h_{R / \mathcal{I}_{\lambda}}(q)=\sum_{\mu \vdash n} f^{\mu} K_{\mu \lambda}(1 / q) q^{n(\lambda)} .
$$

Here, $f^{\mu}$ and $n(\lambda)$ are two well-known parameters associated with partitions ([M]), and $K_{\mu \lambda}(q)$ are the Kostka-Foulkes polynomials we referred to in the introduction ([LS]). The computation of $K_{\mu \lambda}(q)$ is somewhat complicated. This motivates us to use the results of this paper to give a new description of the Hilbert series in the case of hooks.

Let $\lambda=(a \mid b)$ be a hook partition of $n$, and consider the ideal $\mathcal{I}_{\lambda}=\mathcal{M}_{b+1}+\mathcal{E}_{b}$. Since $R / \mathcal{M}_{b+1}$ is a Cohen-Macaulay ring (Corollary 4.1), and the generators $e_{1}\left(x_{1}, \ldots, x_{n}\right), \ldots, e_{b}\left(x_{1}, \ldots, x_{n}\right)$ of $\mathcal{E}_{b}$ form a regular sequence over $R / \mathcal{M}_{b+1}$ (Proposition 4.3), it follows that (see [V] Theorem 4.2.5)

$$
h_{R / \mathcal{I}_{\lambda}}(q)=\prod_{i=1}^{b}\left(1-q^{i}\right) h_{R / \mathcal{M}_{b+1}}(q) .
$$

So we focus on finding $h_{R / \mathcal{M}_{b+1}}(q)$. Recall that $\mathcal{M}_{b+1}$ is generated by all square-free monomials of degree $b+1$ with variables in $\left\{x_{1}, \ldots, x_{n}\right\}$. So each graded piece $\left(R / \mathcal{M}_{b+1}\right)_{s}$ is generated by all monomials of degree $s$, involving $c$ of the $n$ variables with $c \leq b$. There are $\left(\begin{array}{l}n \\ c\end{array}\right)$ ways of choosing $c$ variables from $\left\{x_{1}, \ldots, x_{n}\right\}$. Choose such a monomial, without loss of generality, $x_{1}^{a_{1}} \ldots x_{c}^{a_{c}}$. We need to choose the positive integers $a_{1}, \ldots, a_{c}$ such that $a_{1}+\ldots+a_{c}=s$. There are $\left(\begin{array}{l}s-1 \\ c-1\end{array}\right)$ ways of doing that. So we have

$$
h_{R / \mathcal{M}_{b+1}}(q)=1+\sum_{s=1}^{\infty} \sum_{c=1}^{b}\left(\begin{array}{l}
n \\
c
\end{array}\right)\left(\begin{array}{l}
s-1 \\
c-1
\end{array}\right) q^{s} .
$$

Therefore, by Equation (13) we obtain that $h_{R / \mathcal{I}_{\lambda}}(q)$ equals

$$
\left(1+\sum_{s=1}^{\infty} \sum_{c=1}^{b}\left(\begin{array}{l}
n \\
c
\end{array}\right)\left(\begin{array}{l}
s-1 \\
c-1
\end{array}\right) q^{s}\right) \prod_{i=1}^{b}\left(1-q^{i}\right)=\left(1+\sum_{c=1}^{b}\left(\begin{array}{l}
n \\
c
\end{array}\right) \sum_{s=1}^{\infty}\left(\begin{array}{l}
s-1 \\
c-1
\end{array}\right) q^{s}\right) \prod_{i=1}^{b}\left(1-q^{i}\right) .
$$

On the other hand, it is well known that $\sum_{j=0}^{\infty}\left(\begin{array}{c}i+j \\ i\end{array}\right) q^{j}=\frac{1}{(1-q)^{i+1}}$, ( see ([Wi, page 53, 2.5.7]) ). Hence, we get

$$
\sum_{s=1}^{\infty}\left(\begin{array}{l}
s-1 \\
c-1
\end{array}\right) q^{s}=\frac{q^{c}}{(1-q)^{c}}
$$


We conclude that

$$
\begin{aligned}
h_{R / \mathcal{I}_{\lambda}}(q) & =\prod_{i=1}^{b}\left(1-q^{i}\right) \cdot\left(1+\sum_{c=1}^{b}\left(\begin{array}{l}
n \\
c
\end{array}\right) \frac{q^{c}}{(1-q)^{c}}\right) \\
& =\prod_{i=1}^{b}\left(\frac{1-q^{i}}{1-q}\right) \cdot \sum_{c=0}^{b}\left(\begin{array}{c}
n \\
c
\end{array}\right) q^{c}(1-q)^{b-c}=[b]_{q} ! \sum_{c=0}^{b}\left(\begin{array}{l}
n \\
c
\end{array}\right) q^{c}(1-q)^{b-c},
\end{aligned}
$$

where $[b]_{q} !:=[1]_{q}[2]_{q} \cdots[b]_{q}$ with $[b]_{q}:=1+q+\ldots+q^{b-1}$. We have shown that following proposition holds:

Proposition 5.5. Let $\lambda=(a \mid b)$ be a hook partition of $n$. Then

$$
h_{R / \mathcal{I}_{\lambda}}(q)=[b]_{q} ! \sum_{c=0}^{b}\left(\begin{array}{l}
n \\
c
\end{array}\right) q^{c}(1-q)^{b-c} .
$$

Note that if we set $q=1$ in (14), we find that $\operatorname{dim}_{k}\left(R / \mathcal{I}_{\lambda}\right)=\frac{n !}{(a+1) !}=\frac{n !}{\lambda_{1} !}$ as expected.

Remark 5.6. The formula for Proposition 5.5 has the following nice alternative form, which was suggested by Mark Shimozono.

$$
h_{R / \mathcal{I}_{\lambda}}(q)=[b]_{q} ! \sum_{i=0}^{b} q^{i}\left(\begin{array}{c}
i+a \\
a
\end{array}\right)
$$

\section{Combinatorial Remarks}

As noticed in the introduction, the quotient $R / \mathcal{I}_{\lambda}$ has been extensively studied in algebraic combinatorics [AB, $\mathrm{BG}, \mathrm{GP}]$. Its combinatorial nature arises also in the two following observations on the Poincaré series. We think that they might be useful, in order to understand the behavior of the Poincare series in the case when $\lambda$ is an arbitrary partition.

Remark 6.1 (Recursive nature of the Poincaré series). From Theorem 4.4 it can be seen that the Poincaré series of $\mathcal{I}_{\lambda}$ can be computed recursively. We start with the one-column partition $(0 \mid b)$. In this case the ideal $\mathcal{I}_{\lambda}$ is generated by the first $b+1$ elementary symmetric functions in the variables $x_{1}, \ldots, x_{b+1}$; the quotient $R / \mathcal{I}_{\lambda}$ is known as the coinvariant algebra of the symmetric group, and as $\mathfrak{S}_{b}$-representation is isomorphic to the regular representation (see e.g. $[\mathrm{Hu}]$ ). The graded Poincare series in this case is

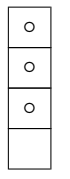

$$
P_{(0 \mid b)}(q, t)=\prod_{k=1}^{b+1}\left(1+q t^{k}\right)
$$

Using Equation (7), by subtracting $P_{(a-1 \mid b)}(q, t)$ from $P_{(a \mid b)}(q, t)$, we get

$$
P_{(a \mid b)}(q, t)=P_{(a-1 \mid b)}(q, t)+\prod_{k=1}^{b}\left(1+q t^{k}\right) \cdot q t^{b+1}\left(\begin{array}{c}
b+a \\
a
\end{array}\right)(1+q t)^{a} .
$$

This recursion allows us to compute the graded Poincaré polynomial of $(a \mid b)$, starting from (15), by adding one cell at a time to the first row of the one-column partition $(0 \mid b)$. For example,

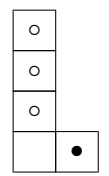

$$
P_{(1 \mid b)}(q, t)=\left(1+q t^{b+1}+q t^{b+1}(b+1)(1+q t)\right) \prod_{k=1}^{b}\left(1+q t^{k}\right) .
$$


Question 6.2. It would be nice to understand what would happen if we added a box not only in the first row, but also in other rows and columns. This would allow the computation of the Poincaré series for any partition.

The second relation we show concerns the single-graded Poincaré series. We compute first its generating function. For simplicity, for any hook $\lambda=(a \mid b)$ we denote by $P(a \mid b)$ the single-graded Poincaré series $P_{R / \mathcal{I}_{(a \mid b)}}(q, 1)$.

Lemma 6.3 (The generating function of the Poincaré series). The generating function of the single-graded Poincaré series is given by

$$
\mathcal{P}=\sum_{a, b \geq 0} P(a \mid b) u^{a} v^{b}=\frac{1}{1-u}\left[\frac{1}{1-(1+q) v}+\frac{q}{1-(1+q)(u+v)}\right] .
$$

Proof. Set $t=1$ in Eq. (7). Then,

$$
P(a \mid b)=(1+q)^{b}+q \sum_{i=0}^{a}\left(\begin{array}{c}
b+i \\
b
\end{array}\right)(1+q)^{b+i} .
$$

We compute the generating functions of the two summands separately. It is clear that

$$
\sum_{a, b \geq 0}(1+q)^{b} u^{a} v^{b}=\frac{1}{1-u} \frac{1}{1-(1+q) v}
$$

On the other hand,

$$
\begin{aligned}
\sum_{a, b \geq 0} \sum_{i=0}^{a}\left(\begin{array}{c}
b+i \\
b
\end{array}\right)(1+q)^{b+i} u^{a} v^{b} & =\sum_{\substack{i, a, b \geq 0 \\
a \geq i}}\left(\begin{array}{c}
b+i \\
b
\end{array}\right)(1+q)^{b+i} u^{a} v^{b}=\sum_{i, b \geq 0}\left(\begin{array}{c}
b+i \\
b
\end{array}\right)(1+q)^{b+i} v^{b} \frac{u^{i}}{1-u} \\
& =\frac{1}{1-u} \sum_{i, b \geq 0}\left(\begin{array}{c}
b+i \\
b
\end{array}\right)(1+q)^{b+i} v^{b} u^{i}=\frac{1}{1-u} \frac{1}{1-(1+q)(u+v)}
\end{aligned}
$$

We have obtained formula (17).

Now it is straightforward to check that the following equation holds

$$
\mathcal{P}=(1-u) \frac{\partial \mathcal{P}}{\partial u}-(1-u) \frac{\partial \mathcal{P}}{\partial v}+\frac{(1+q)}{(1-(1+q) v)^{2}}
$$

By extracting the coefficients of $u^{a} v^{b}$ in both sides of (18) we obtain the following relation

$$
P(a+1 \mid b)-P(a \mid b)=\left(\frac{1+b}{1+a}\right)(P(a \mid b+1)-P(a-1 \mid b+1)),
$$

which holds for all $a>0$, and $b \geq 0$.

\section{References}

[AB] J.-C. Aval and N. Bergeron. Vanishing ideals of lattice diagram determinants, J. Combin. Theory Series A 99 (2002), 244-260.

[BG] N. Bergeron and A. Garsia. On certain spaces of harmonic polynomials, Contemp. Math., 138 (1992), 51-86.

[BH] W. Bruns and J. Herzog, Cohen-Macaulay rings, vol. 39, Cambridge studies in advanced mathematics, revised edition, 1998. 
[DP] C. De Concini and C. Procesi. Symmetric functions, conjugacy classes and the flag variety, Invent. Math. 64 (1981), 203-230.

[GS] D.R. Grayson and M.E. Stillman, Macaulay 2, a software system for research in algebraic geometry, available at http://www.math.uiuc.edu/Macaulay2/.

[ES] D. Eisenbud and D. Saltman, Rank varieties of matrices, Commutative algebra (Berkeley, CA, 1987), 173-212, Math. Sci. Res. Inst. Publ., 15, Springer, New York, 1989.

[ER] J.A. Eagon and V. Reiner, Resolution of Stanley-Reisner rings and Alexander duality, J. Pure Appl. Algebra 130 (1998), no. 3, 265-275.

[GL] A.V. Geramita and A. Lorenzini, Cancellation in resolutions and level algebras, Comm. Algebra 33 (2005), no. 1, 133-158.

[GP] A. Garsia and C. Procesi. On certain graded $S_{n}$-modules and the $q$-Kotska polynomials, Adv. Math. 94 (1992), 82-138.

[HH] J. Herzog and T. Hibi, Cohen-Macaulay polymatroidal ideals, European J. Combin. 27 (2006), no. 4, 513-517.

[HT] J. Herzog and Y. Takayama, Resolutions by mapping cones, The Roos Festschrift volume, 2. Homology Homotopy Appl. 4 (2002), no. 2, part 2, 277-294 (electronic).

[Hu] J. E. Humphreys, Reflection Groups and Coxeter Groups, Cambridge Stud. Adv. Math., no.29, Cambridge Univ. Press, Cambridge, 1990.

[LS] A. Lascoux and M. P. Schützembergerg. Sur une conjecture de H. O. Foulkes, C. R. Acad. Sci. Paris 286 (1978), 323-324.

[M] I.G. Macdonald. Symmetric functions and Hall polynomials, 2nd edition, The Clarendon Press, Oxford University Press, 1995.

[Sc] H. Schenck, Computational algebraic geometry, London Mathematical Society Student Texts, 58. Cambridge University Press, Cambridge, 2003. xiv+193 pp. ISBN: 0-521-82964-X; 0-52153650-2.

[T] T. Tanisaki, Defining ideals of the closure of conjugacy classes and representations of the Weyl groups, Tohoku J. Math. 34 (1982), 575-585.

[V] R. Villarreal, Monomial algebras, Monographs and Textbooks in Pure and Applied Mathematics, 238. Marcel Dekker, Inc., New York, 2001.

[W] J. Weyman, The equations of conjugacy classes of nilpotent matrices, Invent. Math. 98 (1989), no. 2, 229-245.

[Wi] H. Wilf, generatingfunctionology, Second edition. Academic Press, Inc., Boston, MA, 1994. 